Conclusions Colitis reported on CT correlates with endoscopic colitis in only $8 \%$ of patients in this study. Less than $5 \%$ are diagnosed with IBD at 6 months follow up. The correlation improves in younger patients and with shorter interval between CT and endoscopy. One in five patients had completely normal endoscopy and over $90 \%$ had a benign diagnosis. Radiological reporting of fat stranding was an independent risk factor for endoscopic colitis. Anaemia and raised CRP helps identify those at higher risk of malignancy whilst raised CRP alone shows a trend towards identifying true colitis. We conclude that the findings of 'colitis' on CT does not imply IBD in the majority.

\section{PTH-111 IBD NURSE LED RAPID REFERRAL PATHWAY FOR SUSPECTED IBD - IS IT EFFECTIVE?}

1,2Jessica Posso*, 'Vanessa Cambridge, 'David Walker. 'Royal United Hospitals Bath NHS Foundation Trust, Bath, UK; ${ }^{2}$ University of Bristol, Bristol, UK

\subsection{6/gutjnl-2019-BSGAbstracts. 170}

Introduction Recommendations from the UK IBD Standards Group stress the importance of adequate referral pathways in maintaining a high quality of clinical care. In 2016 the gastroenterology department at the Royal United Hospital Bath developed a new rapid referral pathway for suspected inflammatory bowel disease (IBD) patients in order to improve patient care. All GP referrals for suspected IBD were evaluated by a gastroenterology consultant. The clinical information together with any available blood and stool results were reviewed. Symptomatic patients with a suspected new diagnosis of colitis were triaged to an urgent IBD nurse appointment (within 4 weeks) and if appropriate, an urgent flexible sigmoidoscopy with the IBD nurse was offered later the same day. Those patients with a new diagnosis of IBD were given treatment and educational information post procedure. The primary aim of the study was to assess the effectiveness of the new rapid referral pathway and to see if it could be improved further.

Methods A retrospective review was performed of all suspected IBD referrals that had been triaged to an urgent IBD nurse appointment over a 1 year period. For each case identified, a structured proforma review was performed using the original GP referral, the patient's medical notes, blood profile, stool analysis, endoscopy report and histology.

Results 30 patients were referred via the rapid access pathway over a 1 year period. All 30 patients had a flexible sigmoidoscopy with biopsies performed. $30 \%(9 / 30)$ had a new diagnosis of IBD [8 UC, 1 IBD-U], 3\% (1/30) had diverticulitis and $67 \%(20 / 30)$ had a normal procedure. In $93 \%$ of cases bloods including a FBC \& CRP had been performed at the time of referral. However stool microscopy was only performed in $60 \%$ of cases and a faecal calprotectin (FC) in $37 \% .83 \%$ of patients were seen within 4 weeks and all patients were seen within 6 weeks.

Conclusions At the Royal United Hospital Bath, there has been a significant year on year increase in the number of 2WW referrals which has negatively impacted on the ability to see suspected IBD patients within the recommended 4 week period. The new IBD nurse led rapid access pathway resulted in $83 \%$ of suspected IBD patients being reviewed within 4 weeks and all suspected IBD patients being reviewed within 6 weeks. Interestingly, only $30 \%$ of patients were found to have a new diagnosis of IBD. The study showed that FC testing was only happening in a minority of cases. The importance of FC testing has since been highlighted at educational meetings with local GPs and a more rigid IBD referral proforma has been developed. This study shows that an IBD nurse led rapid referral pathway can improve the patient experience by facilitating a quicker diagnosis of IBD and enhances continuity of care.

\section{PTH-112 EFFECT OF CO-MORBIDITIES IN CROHN'S DISEASE ASSOCIATED URINARY METABOLIC PROFILES}

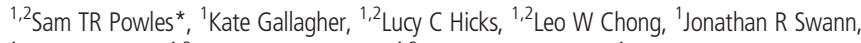
${ }^{1}$ Elaine Holmes, ${ }^{1,2}$ Horace RT Williams, ${ }^{1,2}$ Timothy R Orchard. ${ }^{1}$ Imperial College London, London, UK; ${ }^{2}$ Imperial College Healthcare NHS Trust, London, UK

\subsection{6/gutjnl-2019-BSGAbstracts. 17}

Introduction Distinct metabolic signatures have been detected in urine that differentiate Crohn's disease (CD) from controls in multiple studies, with consistent discriminatory metabolites derived from bacteria and co-bacterial pathways (Williams, 2009. AJG). Multiple other diseases have also been found to affect the urinary metabolome, and many of these relate to changes in bacterial associated metabolites (Lu, 2013. Front. Med.). This study aimed to examine a real life cohort of CD patients, and so included patients with other co-morbidities, to examine if the same metabolite changes were present, and if these patients could be distinguished from controls despite the presence of co-morbidities.

Methods Nuclear magnetic resonance ( $\left.\mathrm{H}^{1} \mathrm{NMR}\right)$ spectroscopy was used to acquire urinary metabolic data from $74 \mathrm{CD}$ patients and 100 controls. 19 of the CD group and 48 of the controls had at least one significant co-morbidity (diabetes, asthma, hypertension). Multivariate analysis was performed using OPLSDA. Univariate analysis was also performed to assess whether bacterial associated metabolites, as demonstrated in previous studies (Williams, 2009), were significantly different in CD patients compared to controls. These metabolites were Hippurate, Alanine, Citrate, P-Cresol, Phenyacetylglutamine (PAGn), and Dimethylglycine (DMG). 


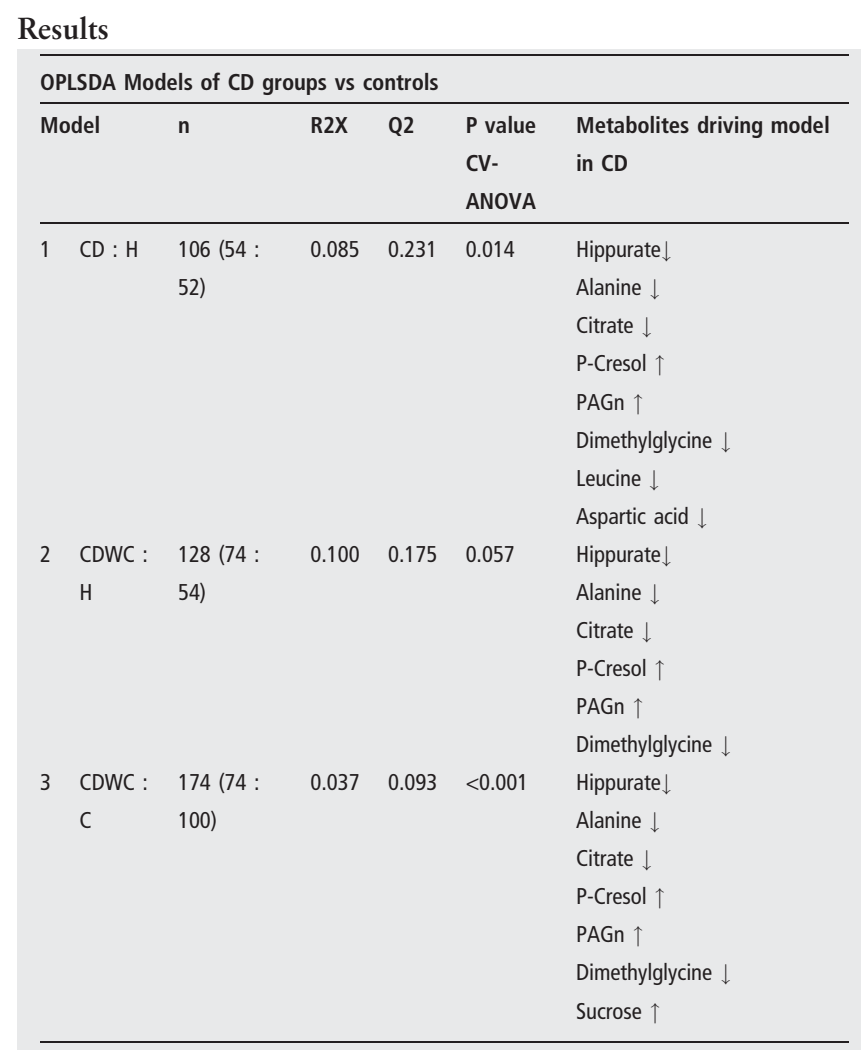

OPLSDA analysis showed statistically significant separation between $C D$ patients and controls irrespective of the presence of comorbidities. Model 1 compares CD patients to healthy controls $(\mathrm{H})$. Models 2 and 3 include $\mathrm{CD}$ patients with at least one other co-morbidity (CDWC), and Model 3 includes non-CD patients with another co-morbidity in the control group (C).

Univariate analysis showed that the bacterial associated metabolites Hippurate, Citrate, P-Cresol, DMG, and PAGn changed with statistical significance between $\mathrm{CD}$ groups and controls irrespective of the presence of co-morbidities.

Conclusions The pattern of change in discriminating metabolites appear to be preserved in models separating CD from controls when patients with co-morbidities are included in the models, and these groups can be significantly separated with multivariate analysis. It is likely the effect of microbial disturbance is still measurable with this technique in a real world cohort.

\section{PTH-113 EFFECT OF ETHNICITY ON THE FAECAL WATER METABOLIC PROFILES IN CROHN'S DISEASE}

1,2Sam TR Powles*, 1,2 Leo W Chong, 'Kate I Gallagher, 1,2Lucy C Hicks, 'Jonathan R Swann, 'Elaine Holmes, ${ }^{1,2}$ Horace RT Williams, ${ }^{1,2}$ Timothy R Orchard. ${ }^{1} /$ mperial College London, London, UK; ${ }^{2}$ Imperial College Healthcare NHS Trust, London, UK

\subsection{6/gutjnl-2019-BSGAbstracts.172}

Introduction South Asians (SA) with Crohn's disease (CD) display a different disease phenotype and course (Walker, 2011. AJG.), as well as a different urinary metabolic profile (Marchesi, 2008. J. Proteome Res.). Metabolic profiling of faecal water has distinguished CD from controls previously, but only in Caucasian (Cau) cohorts, characterised by a reduction in short chained fatty acids. In this study, we aimed to compare the metabolic profiles of faecal water in CD patients and healthy controls $(\mathrm{H})$ from Caucasian and South Asian backgrounds.

Methods Samples from 28 CD patients (15 Cau and 13 SA) and 44 healthy controls (20 Cau and 24 SA) were analysed by $\mathrm{H}^{1} \mathrm{NMR}$ spectroscopy. Data was assessed using orthogonal partial least squares discriminant analysis (OPLSDA). Hypothesis-led univariate analysis was also performed using metabolites that have been previously shown to distinguish CD from controls (Marchesi, 2008). These metabolites were as follows: Acetate, Butyrate, Propionate, Lactate, Methylamine, Glutamine, Alanine, Taurine, Valine, and 2-Hydroxybutyrate (2HIB).

Results Multivariate analysis (table 1) demonstrated that the faecal metabolites driving the separation between CD and controls were similar regardless of ethnicity, although statistically significant separation could not be achieved by multivariate analysis in the smallest group (CD v $\mathrm{H}$ in Caucasians).

Univariate analysis demonstrated a statistically significant reduction in Butyrate, Acetate, 2-HIB, and Methylamine ( $\mathrm{p}<$ 0.05) in Cau CD patients compared to controls consistent with previous studies, but only a reduction in 2-HIB and Methylamine were seen with statistical significance in SA patients. When directly comparing Cau and SA patients with $\mathrm{CD}$, Butyrate and 2-HIB were significantly different $(\mathrm{p}=$ 0.034 and 0.035 respectively) between these ethnic groups.

Conclusions Despite observing consistent changes in the metabolites driving separation between Caucasian and SA patients compared to controls, univariate analysis reveals differences relating to ethnicity, showing that ethnicity influences

\begin{tabular}{|c|c|c|c|c|c|}
\hline $\begin{array}{l}\text { Model } \\
\text { OPLSDA }\end{array}$ & $n$ & $R 2 X$ & Q2 & $\begin{array}{l}\text { P value } \\
\text { CV- } \\
\text { ANOVA }\end{array}$ & $\begin{array}{l}\text { Metabolites } \\
\text { driving separation in } \\
\text { CD }\end{array}$ \\
\hline $\begin{array}{l}\mathrm{CD}: \mathrm{HC} \\
\text { All }\end{array}$ & $\begin{array}{l}72 \\
(28: 44)\end{array}$ & 0.145 & 0.197 & $<0.001$ & $\begin{array}{l}\text { Acetate } \downarrow \\
\text { Butyrate } \downarrow \\
\text { Glutamine } \downarrow \\
\text { Alanine } \downarrow \\
\text { Creatine } \downarrow \\
\text { Tryptophan } \downarrow \\
\text { 2-HIB } \downarrow \\
\text { Methylamine } \downarrow\end{array}$ \\
\hline $\begin{array}{l}\mathrm{CD}: \mathrm{H} \\
\text { Caucasian }\end{array}$ & $\begin{array}{l}35 \\
(15: 20)\end{array}$ & 0.122 & 0.126 & 0.117 & $\begin{array}{l}\text { Acetate } \downarrow \\
\text { Butyrate } \downarrow \\
\text { Glutamine } \downarrow \\
\text { Alanine } \downarrow \\
\text { Creatine } \downarrow \\
\text { Tryptophan } \downarrow \\
\text { 2-HIB } \downarrow \\
\text { Methylamine } \downarrow\end{array}$ \\
\hline $\begin{array}{l}\mathrm{CD}: \mathrm{H} \\
\text { South } \\
\text { Asians }\end{array}$ & $\begin{array}{l}37 \\
(13: 24)\end{array}$ & 0.196 & 0.290 & 0.024 & $\begin{array}{l}\text { Acetate } \downarrow \\
\text { Butyrate } \downarrow \\
\text { Glutamine } \downarrow \\
\text { Alanine } \downarrow \\
\text { Creatine } \downarrow \\
\text { Tryptophan } \downarrow \\
\text { 2-HIB } \downarrow \\
\text { Methylamine } \downarrow\end{array}$ \\
\hline
\end{tabular}

